\title{
Developmental pruning of early-stage myelin segments during CNS myelination in vivo
}

Cell Research (2013) 23:962-964. doi:10.1038/cr.2013.62; published online 7 May 2013

\section{Dear Editor,}

Axon-wrapping myelin sheath is essential for proper functioning of the nervous system. Myelin is formed from membranous sheets that are elaborated by oligodendrocytes (OLs) in the central nervous system (CNS). Although much is known about the development and migration of $\mathrm{OL}$ and its progenitor cell in vitro and in vivo [1-3], how CNS myelin is dynamically formed through OL processes is still unclear $[1,3]$. To examine the process of CNS myelination in vivo, we performed longterm time-lapse confocal imaging of the same OLs in the spinal cord of intact zebrafish larvae during 2-7 days post-fertilization (dpf) with intervals of 2-12 h. Individual OLs were mosaically labeled by membrane-tethered red fluorescent protein (mRFP) under the control of zebrafish Sox10 regulatory DNA [2]. We find that, besides extension of some early-stage axon-wrapping myelin segments, 28\% segments undergo pruning in an axon diameter-correlated manner during early development. Downregulating neuregulin-ErbB signaling impairs the pruning but not extension. Thus, our study reveals earlystage myelin pruning as a novel process during myelination.

First, we found that, in zebrafish larvae, OLs initially emanated numerous processes (Figure $1 \mathrm{~A}_{1}$ ), and then synchronously formed multiple early-stage myelin segments at $80.6 \pm 9.7 \mathrm{~h}$ post-fertilization (hpf; Figure $1 \mathrm{~A}_{2}$ and $1 \mathrm{~B})$. To simultaneously visualize axons, transgenic $\operatorname{Tg}(H u C: G F P)$ zebrafish, in which some axons in the spinal cord express green fluorescent protein (GFP), were used (Supplementary information, Figure S1). To quantify the myelination process, we measured the total number of early-stage myelin segments in individual OLs and the length of each segment at each time point. Interestingly, the total number of segments gradually decreased from $12.5 \pm 1.1$ at the initiation time point to 8.3 \pm 0.8 after 4 days $\left(P<0.01\right.$; Figure $1 \mathrm{~A}_{2}-1 \mathrm{~A}_{6}$ and $\left.\mathrm{C}\right)$. This decrease was mainly due to the fact that, after the initiation of myelin segments, although many of them continued to extend in their length ("extending segment"), 28.6 $\pm 3.4 \%$ of these early-stage myelin segments underwent retraction and final pruning in 25 out of 27 OLs examined ("pruning segment"; Figure 1D and 1E). The mean lifespan of pruning segments was about $35.8 \pm 2.2 \mathrm{~h}$, and positively correlated with their initial length $(P<0.0001$; Supplementary information, Figure S2). These results raise the possibility that, besides myelin extension, myelin pruning may be another important process during myelination.

Next, we found that this early-stage myelin pruning preferred to occur at small but not large axons. In the spinal cord of zebrafish, the Mauthner ("M") cell axon, the largest one in the animal, is the first to be myelinated [4]. Some OLs initially formed myelin segments on both Maxons and other small axons (Figure $1 \mathrm{~F}$ and Supplementary information, Figure S3). In those OLs, early-stage myelin segments on $\mathrm{M}$-axons never underwent pruning ( 0 out of 15 ; "M-axon's myelin segment"; Figure $1 \mathrm{~F}$ and $1 \mathrm{G}$, and Supplementary information, Figure S3), whereas segments on small axons by the same OL ("small axon's myelin segment of M-axon-wrapping OL") exhibited faster pruning with a higher probability in comparison with the segments of OLs which did not wrap M-axons ("small axon's myelin segment of non-M-axon-wrapping OL"; Figure $1 \mathrm{G}$ and $1 \mathrm{H}$ ).

The membrane-bound neuregulin1 (NRG1) is an axonal ligand for glial ErbB receptors, and NRG1-ErbB signaling is required for myelination in both the peripheral and central nervous systems [5-8]. To examine whether this signaling pathway has a role in the early-stage myelin pruning, we first used two previously characterized morpholino oligomers (MOs) to knock down the expression of NRG1 in zebrafish larvae. In NRG1 knockdown larvae (morphants), the number of initial myelin segments was not significantly different from that in wildtype larvae (Figure 1I and Supplementary information, Figure S4A and S4B). However, the probability of earlystage myelin segment pruning in NRG1 morphants was markedly decreased (NRG1 morphant vs Control: $11.9 \pm$ $2.0 \%$ vs $28.6 \pm 3.4 \%, P<0.01$; Figure $1 \mathrm{~J}$ ), and the process of residual pruning events was also slowed down 

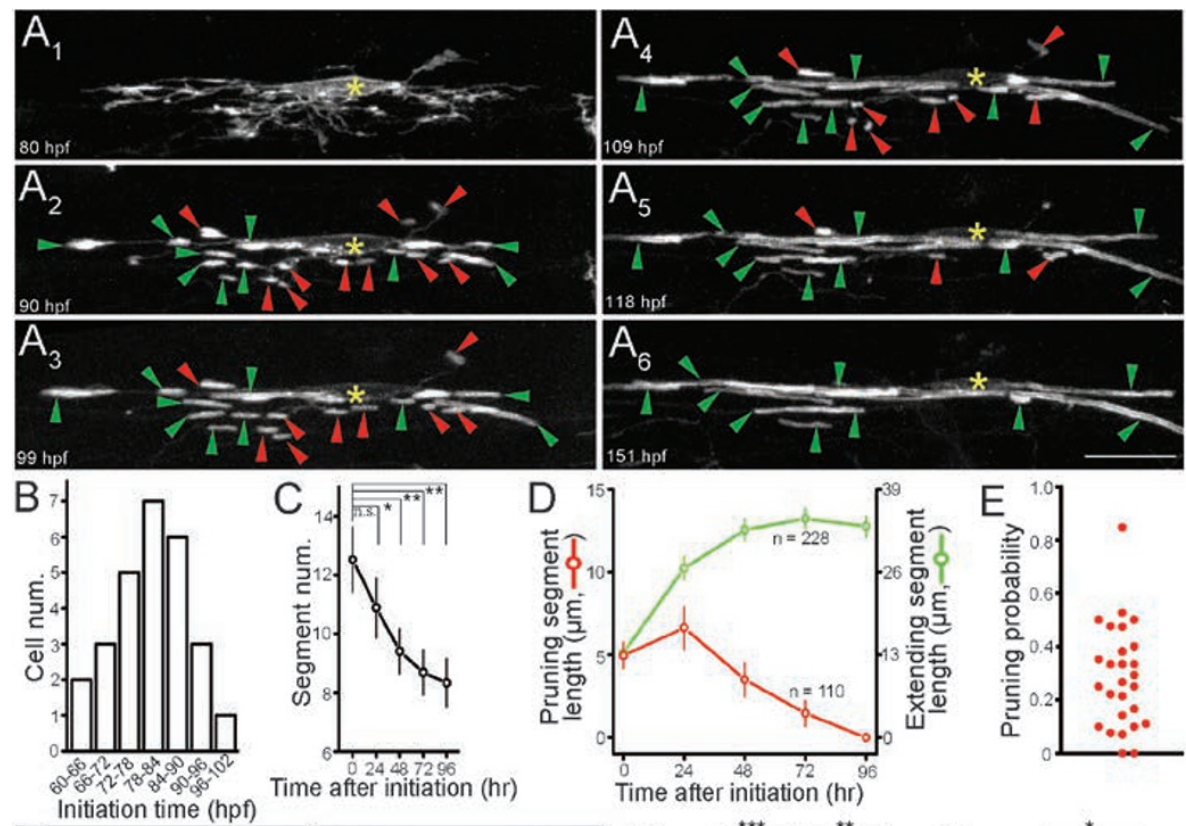

Time after initiation (hr)
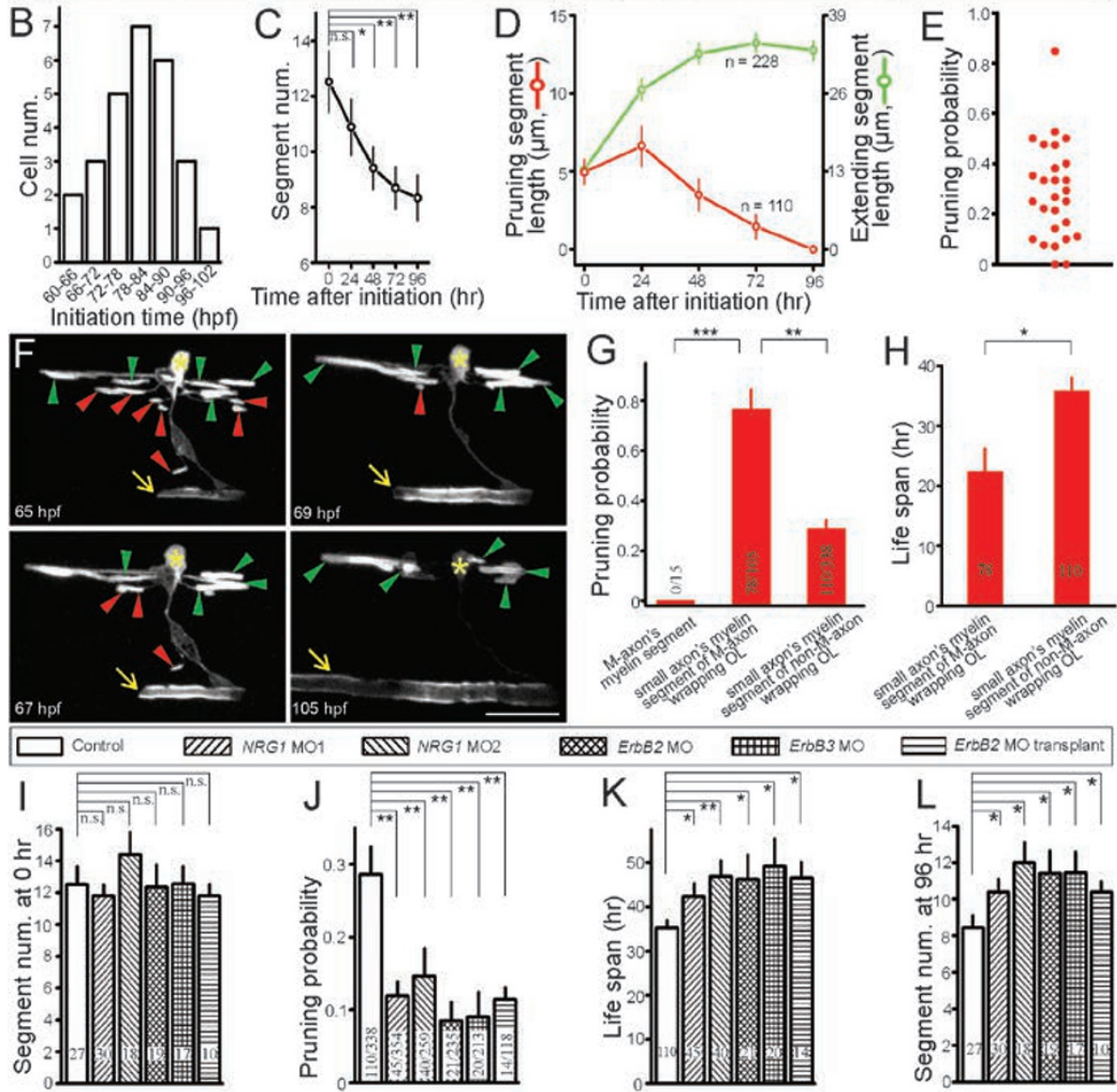

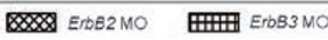

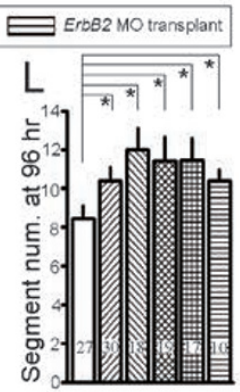

Figure 1 Pruning of early-stage myelin segments during oligodendrocyte myelination. $\left(\mathbf{A}_{1}-\mathbf{A}_{6}\right)$ Time-lapse images of an oligodendrocyte $(\mathrm{OL})$ in the spinal cord of a zebrafish larva. Lateral views with dorsal up and anterior left. Asterisk, OL soma; Green arrowhead, extending myelin segment; Red arrowhead, pruning myelin segments; hpf, hours post-fertilization. Scale: $20 \mu \mathrm{m}$. (B) Initiation time of myelination. Data were obtained from 27 OLs in 21 animals. (C) Time course showing change in the number of myelin segment after initiation. Same data set with B was used. hr, hour. (D) Time course showing changes in the mean length of pruning (red) and extending (green) segments after initiation. The numbers indicate the numbers of myelin segments examined in 27 OLs. (E) Pruning probability of early-stage myelin segments in individual OLs $(n=27)$. Same data set with B was used. The pruning probability was calculated by dividing the number of pruning segments after $96 \mathrm{~h}$ with the total segment number at the initiation time. (F-H) Axon diameter-dependency of myelin segment pruning. (F) Time-lapse images of an OL, one process of which wrapped Mauthner cell axon (arrow). Asterisk, OL soma; Green arrowhead, extending myelin segment; Red arrowhead, pruning myelin segments. Scale, $20 \mu \mathrm{m}$. (G) Pruning probability of different types of myelin segments. Number/number, pruning segment number/total segment number. (H) Lifespan of pruning myelin segments of M-axon- and non-M-axon-wrapping OLs. The numbers on bars represent the numbers of pruning segments examined. (I-L) NRG1-ErbB signaling is required for myelin segment pruning. The data obtained from OLs without contacting Mauthner cell axons were analyzed. (I) Mean number of total myelin segments per OLs at the myelination initiation timepoint. The numbers on bars represent the numbers of OLs examined. (J) Pruning probability of myelin segments. Number/number, pruning segment number/total segment number. (K) Life span of pruning myelin segments. (L) Mean number of total myelin segments per OLs $96 \mathrm{~h}$ after myelination initiation. n.s., no significance; ${ }^{*} P<0.05$; ${ }^{* *} P<0.01$; ${ }^{* *} P<0.001$ (unpaired Student's $t$-test and one-way ANOVA). Error bars represent mean \pm SEM. 
$(P<0.05$; Figure $1 \mathrm{~K})$, leading to an increase in the total number of myelin segments examined $96 \mathrm{~h}$ later $(P<0.05$; Figure 1L). Meanwhile, the growth rate of extending myelin segments was not significantly affected (Supplementary information, Figure S4E). As a result, the total length of segments in individual OLs was increased in NRG1 morphants $(P<0.05$; Supplementary information, Figure S4F). Similar results were observed when the expression of ErbB2 or ErbB3 was downregulated (Figure 1I-1L and Supplementary information, Figure S4). These results suggest that NRG1-ErbB signaling is important for the early-stage myelin segment pruning.

To examine whether ErbB expressed in oligodendrocytes is required, we transplanted some cells at the blastula stage from donor transgenic $\operatorname{Tg}$ (Sox 10:mRFP) embryos, which were injected with ErbB2 morpholino, into wild-type host embryos. Thus, in chimera embryos, RFP-positive oligodendrocytes were originated from ErbB2 morphants and carried deficiency in ErbB2. We found that the RFP-positive oligodendrocytes in chimeras initially formed numerous early-stage myelin segments with a number comparable to that under normal condition ("ErbB2 MO transplant", Figure 1I). However, only few of those segments underwent pruning during development, similar to what we observed in ErbB2 morphants (Figure 1J and Supplementary information, Figure S5). This indicates that ErbB2 in oligodendrocytes is required.

In the nervous system, pruning of neural processes and synapses is a common aspect during development, and ensures the establishment of proper neural connections [9]. Here, we find that early-formed axon-wrapping myelin segments also undergo extensive pruning, which may accompany axon pruning during development. Furthermore, segments wrapping larger axons will win and ones wrapping smaller axons will lose, raising the possibility that myelin pruning exhibits a competitive property. This may explain why large axons recruit glial wrapping early and receive more myelin layers than small axons $[1,4,10]$. During the pruning process, NRG-ErbB signaling may serve as a competitive signal at extending myelin segments or a pruning signal at pruning segments. It is of interest to discriminate these possibilities in the future.

\section{Acknowledgments}

We thank Dr B Appel at University of Colorado School of Medicine for providing the Sox10:mRFP plasmid. This work was supported by grants to $\mathrm{CH}$ from National Key Basic Research Program (2011CB504401), National Natural Science Foundation (31130024, 31070922), and to JLD. from 973 Program (2011CBA00400, 2012CB945101).

\author{
Peng Liu ${ }^{1}$, Jiu-lin $\mathrm{Du}^{2}$, Cheng $\mathrm{He}^{1}$ \\ ${ }^{I}$ Department of Neurobiology and Key Laboratory of Molecular Neurobi- \\ ology of Ministry of Education, Second Military Medical University, 800 \\ Xiangyin Road, Shanghai 200433, China; ${ }^{2}$ Institute of Neuroscience and \\ State Key Laboratory of Neuroscience, Shanghai Institutes for Biological \\ Sciences, Chinese Academy of Sciences, 320 Yue-Yang Road, Shanghai \\ 200031, China \\ Correspondence: Jiu-lin $\mathrm{Du}^{\mathrm{a}}$, Cheng $\mathrm{He}^{\mathrm{b}}$ \\ ${ }^{\mathrm{a}}$ Tel: +86-21-54921825; Fax: +86-21-54921735 \\ E-mail: forestdu@ion.ac.cn \\ ${ }^{\mathrm{b}}$ Tel: +86-21-65515200; Fax: +86-21-65492132 \\ E-mail: chenghe@smmu.edu.cn
}

\section{References}

1 Nave KA, Trapp BD. Annu Rev Neurosci 2008; 31:535-561.

2 Kirby BB, Takada N, Latimer AJ, et al. Nat Neurosci 2006; 9:15061511.

3 Watkins TA, Emery B, Mulinyawe S, et al. Neuron 2008; 60:555-569.

4 Almeida RG, Czopka T, Ffrench-Constant C, et al. Development 2011; 138:4443-4450.

5 Michailov GV. Science 2004; 304:700-703.

6 Makinodan M, Rosen KM, Ito S, et al. Science 2012; 337:1357-1360.

7 Lyons DA, Pogoda H-M, Voas MG, et al. Current Biol 2005; 15:513524

8 Brinkmann BG, Agarwal A, Sereda MW, et al. Neuron 2008; 59:581595 .

9 Luo L, O’Leary DD. Annu Rev Neurosci 2005; 28:127-156.

10 Lee S, Leach MK, Redmond SA, et al. Nat Methods 2012; 9:917-922.

(Supplementary information is linked to the online version of the paper on the Cell Research website.) 